Minireview

\title{
Photophosphorylation and the chemiosmotic perspective
}

\author{
André T. Jagendorf \\ Plant Biology Department, Cornell University, Ithaca, NY 14853, USA (e-mail: atj1 @ cornell.edu; fax: +1-607- \\ 255-5407)
}

Received 24 October 2001; accepted in revised form 29 November 2001

Key words: D. Arnon, M. Avron, ATPase, $\mathrm{CF}_{1}$, chemiosmotic hypothesis, chloroplasts, coupling factor, A. Frenkel, G. Hind, A. Jagendorf, R. McCarty, P. Mitchell, photophosphorylation

\begin{abstract}
Photophosphorylation was discovered in chloroplasts by D. Arnon and coworkers, and in bacterial 'chromatophores' (intercytoplasmic membranes) by A. Frenkel. Initial low rates were amplified by adding electron-carrying compounds such as FMN, later shown to support the 'pseudocyclic' electron flow. ATP synthesis, and coupling to electron flow, was detected accompanying linear electron flow from $\mathrm{H}_{2} \mathrm{O}$ to either $\mathrm{NADP}^{+}$or ferricyanide. Another pattern of electron flow supporting photophosphorylation was that of a cycle around Photosystem I (PS I). Isolation and analysis of the ATP synthase showed, as with mitochondrial and bacterial analogues, an intrinsic membrane complex $\left(\mathrm{CF}_{0}\right)$ and an extrinsic complex $\left(\mathrm{CF}_{1}\right) . \mathrm{CF}_{1}$ is a latent ATPase, activated additively by the high-energy state of the thylakoids, and by reduction of a disulfide bond on the gamma subunit. Once reduced, ATP synthesis occurs at lower energy levels. The search for an 'intermediate' linking electron flow and ATP synthesis led to the discovery of post-illumination ATP synthesis by thylakoids, where turnover occurs in the dark. Once interpreted by P.Mitchell's chemiosmotic hypothesis, this led to the discovery of light-driven proton uptake into the thylakoid lumen, with accompanying $\mathrm{Cl}^{-}$intake and $\mathrm{Mg}^{2+}$ and $\mathrm{K}^{+}$output. Chemiosmosis was confirmed in several ways, including ATP synthesis in the dark due to an acid-to-base transition of thylakoids, and photophosphorylation accomplished in artificial lipid vesicles containing both the proton-pumping bacterial rhodopsin and a mitochondrial ATPase complex. The now generally accepted chemiosmotic interpretation is able to clarify some other aspects of photosynthesis as well.
\end{abstract}

Abbreviations: DCCD - dicylohexylcarbodiimide; DNP - dinitrophenol; DTT - dithiothreitol; EDTA - ethylene diamine tetraacetic acid; FMN - flavine mono nucleotide; NADP - nicotinamide adenine dinucleotide phosphate; PS I - Photosystem I; SDS - sodium dodecyl sulfate

\section{Discovery}

It was suggested early (Ruben 1943; Emerson et al. 1944) that phosphate bond energy could be an intermediate in photosynthesis. Several groups found indications of photophosphorylation in plant cells: either conversion of cell inorganic phosphate to organic forms (Kandler 1950; Simonis and Grube 1952) or uptake of phosphate and conversion to polyphosphate (Gest and Kamen 1948; Wassink 1957), or actual formation of $\mathrm{AT}^{32} \mathrm{P}$ in light (Strehler 1953; Schwink 1956). Another useful criterion was the up- take of glucose and its conversion to starch in light but not in the dark, requiring at least one ATP (Maclachlan and Porter 1959). In these and many other studies, dinitrophenol (DNP), which uncouples respiration, failed to prevent the light-induced reactions.

Deeper insight into the mechanism for ATP synthesis depended on demonstrating the reaction with cell-free organelles (this had been done for oxidative phosphorylation in homogenates in 1937; and with mitochondria identified as such in 1949). This was accomplished with chloroplasts by Arnon et al. (1954), 'chromatophores' (intercytoplasmic membranes) from 


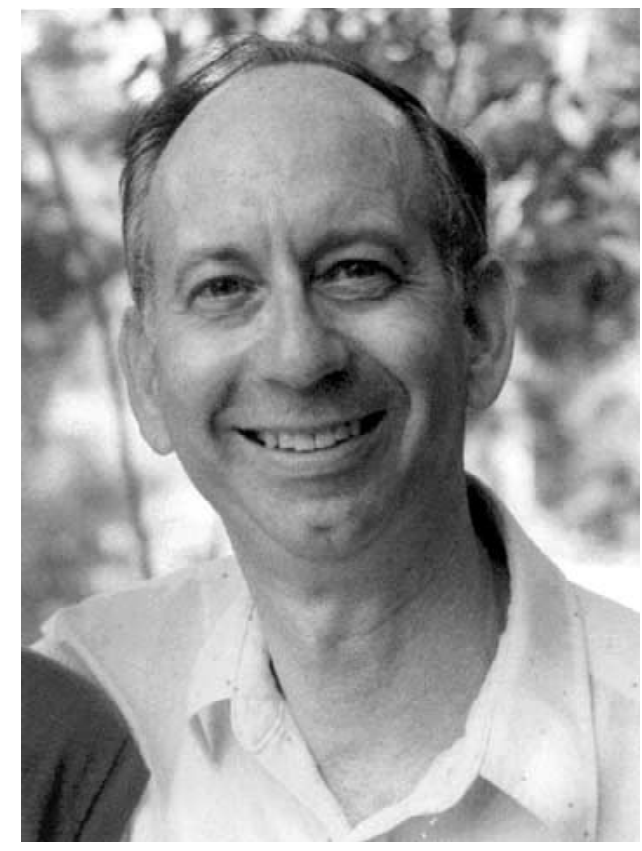

Figure 1. Mordhay Avron.

Rhodospirillum rubrum by Frenkel (1954), and from the anaerobic bacterium Chromatium by Williams (1956). With the 'chromatophores' from both bacteria, it was clear that a cyclic electron flow not involving net oxidation of any substrate supported the ATP synthesis. (A photograph of Daniel Arnon is shown in the article by R. Porra in this issue.)

With isolated chloroplasts (actually, thylakoids) as well, the first discovered ATP synthesis seemed to be supported by a cyclic electron flow pattern. The original rates were at the level of $3 \mu \mathrm{mol} \mathrm{ATP} / \mathrm{mg} \mathrm{Chl} / \mathrm{h}$. However adding FMN or menadione as 'cofactors' permitted rates to be raised to almost $500 \mu \mathrm{mol} / \mathrm{mg}$ $\mathrm{Chl} / \mathrm{h}$ (Allen et al. 1958) and, as in bacterial extracts, methyl phenazinium sulfate (PMS) brought rates up to 900 (Avron and Jagendorf 1958; see Figure 1 for a photograph of Mordhay Avron).

A little later, it was found that linear (uphill) electron flow from water as donor to added $\mathrm{NADP}^{+}$, yielding $\mathrm{O}_{2}$ as a waste product, supported ATP synthesis (Arnon et al. 1958a). This also occurred using nonphysiological electron acceptors such as potassium ferricyanide, although not with trichlorophenol indophenol (Avron et al. 1958).

A variant of this pattern was found in which the added electron acceptor reduces the $\mathrm{O}_{2}$ that had been evolved, that is, a net $\mathrm{O}_{2}$ exchange reaction which can also support ATP synthesis. This is similar to the Mehler reaction (Mehler 1951; see Heber, this issue, for further information), in which $\mathrm{O}_{2}$ is reduced to $\mathrm{H}_{2} \mathrm{O}_{2}$. By a number of criteria [see Jagendorf (1962) for a full discussion], ATP synthesis by FMN, menadione, and many other 'cofactors' arouses this sort of $\mathrm{O}_{2}$ exchange pattern. This sequence was renamed 'psuedocyclic photophosphorylation' (Arnon et al. 1961).

\section{Coupling, and uncouplers}

It had been known earlier (Lardy and Wellman 1952) that electron flow in mitochondria depends almost entirely on added ADP and $\mathrm{P}_{\mathrm{i}}$, permitting simultaneous ATP synthesis. A similar phenomenon, although not so dramatic, was found for linear electron flow from $\mathrm{H}_{2} \mathrm{O}$ to $\mathrm{NADP}^{+}$(Arnon et al. 1958b) and to ferricyanide (Avron et al. 1958).

Uncoupling of mitochondrial oxidation by DNP was demonstrated even earlier (Loomis and Lipmann, 1948). For chloroplasts, ammonium chloride was the first uncoupler found (Krogmann et al. 1959). This was followed very soon by the demonstration of uncoupling by a variety of organic amines (Good 1960). Thus, by 1959 , the bioenergetics of chloroplasts had achieved the position of mitochondrial research in 1952.

\section{$\mathrm{CF}_{1}$ and $\mathrm{CF}_{\mathrm{o}}$}

Discovery of the enzymatic machinery for ATP synthesis similarly benefited from prior work with mitochondria. Efraim Racker (see N. Nelson in this issue for a photograph of Racker) and others, extracted proteins from mitochondria so that oxidative phosphorylation was eliminated, and added them back to restore some phosphorylation (see Racker 1970). These were called coupling factors, and also had ATPase activity. The first demonstration of an extractable coupling factor for chloroplasts was made by Avron (1963), using thylakoids uncoupled by treatment with ethylene diamine tetraacetic acid, EDTA (Jagendorf and Smith 1962). It was nicknamed $\mathrm{CF}_{1}$ (for Chloroplast $F_{1}$ ), to distinguish it from the analogous mitochondrial $F_{1}$ (for factor 1). Subsequently, Vambutas and Racker (1965) purified a $\mathrm{Ca}^{2+}$-requiring ATPase from chloroplasts, which was latent until activated by trypsin. It could restore photophosphorylation to depleted thylakoids. 
It was found that the EDTA treatment releases only a fraction (no more than $70 \%$ ) of the thylakoid's $\mathrm{CF}_{1}$. Chaotropes such as silicotungstate (Lien and Racker 1971) or sodium bromide (Kamienietzky and Nelson 1975) are needed to remove all of it. Reconstitution consists of incubating depleted particles and $\mathrm{CF}_{1}$ together with $\mathrm{Mg}^{2+}$ or other cations, to permit their close association (Telfer et al. 1980).

$\mathrm{CF}_{1}$ was purified and its molecular mass was estimated as $325000 \mathrm{Da}$ (Farron 1970), but improved techniques later established its $\mathrm{Mr}$ value as 400000 (Moroney et al. 1983). Gel electrophoresis in sodium dodecyl sulfate, SDS (Racker et al. 1972) revealed five kinds of subunits, designated $\alpha$ to $\epsilon$ from largest (mass ca. $59 \mathrm{kDa}$ ) to smallest (mass ca. 13 000). Later work found a stoichiometry of $3: 3: 1: 1: 1$. Nucleotide binding sites were found on the $\alpha$ and $\beta$ subunits. Six nucleotide binding sites have been defined (see Hightower and McCarty 1996). Catalysis occurs on the $\alpha$ subunit, or between the $\alpha$ and $\beta$ subunits. The single $\gamma$ subunit must be part of the machinery keeping protons from leaking out of the membrane (Moroney and McCarty 1982; see below), and $\epsilon$ is an inhibitor of ATP hydrolysis or synthesis in some of its configurations (Richter et al. 1984). Roles of the subunits are discussed more fully in Futai et al. (1989) and Jagendorf et al. (1991).

The complete apparatus for synthesis of ATP requires the membrane components to which $\mathrm{CF}_{1}$ attaches. This was designated $\mathrm{CF}_{\mathrm{o}}$ by analogy with the mitochondrial intrinsic membrane protein, which had been called $F_{o}$ because it bound added oligomycin. The first approaches led to isolation of an active $\mathrm{CF}_{1} \mathrm{CF}_{\mathrm{O}}$ complex (Carmeli and Racker 1973); later work permitted isolation of $\mathrm{CF}_{\mathrm{o}}$ by itself (see Sebald and Hoppe 1981). Current ideas on the structure of $\mathrm{CF}_{\mathrm{o}}$ give it four kinds of subunits, numbered I through IV. Subunit III, the smallest (about $8 \mathrm{kDa}$ ), present as a ring of 6-12 copies, binds the energy transfer inhibitor DCCD (dicyclohexylcarbodiimide) (Nelson et al. 1977) and is the pore through which protons move (see below).

In the early days, thylakoids were declared to have neither ATPase nor a Pi/ATP exchange reaction (Avron and Jagendorf 1959). However, by using high concentrations of sulfhydryl reagents in the presence of light, thylakoids were found to have a strong $\mathrm{Mg}^{2+}$ dependent ATPase (Petrack and Lipmann 1961). Subsequent work by a number of groups showed that the thylakoid ATPase is latent in the dark. Activation occurs in two steps: a rapid initial activation by the high-energy state (causing release of a tightly bound inhibitory ADP, and loosening of the $\epsilon$ subunit), and a slower further activation by reduction of a disulfide bond on the $\gamma$ subunit (see BakkerGrunwald, 1977; McCarty 1992). The rate of ATP hydrolysis depends on sufficient high-energy state to be (and remain) active, but excess levels are inhibited by a feed-back mechanism since ATP hydrolysis builds up the energetic state. Low concentrations of an uncoupler will relieve inhibition due to excess highenergy state. Higher concentrations inhibit ATPase by causing the protonmotive force to be so low it can no longer maintain $\mathrm{CF}_{1}$ in an active state.

These characteristics mean that, in bright sunlight in vivo, back pressure from the thylakoids' very highenergy state prevents ATP hydrolysis. But also, in the dark in vivo, ATP is not hydrolyzed because the $\mathrm{CF}_{1}$ is not active. Their stringency prevents futile cycles of ATP synthesis and hydrolysis.

Activation of thylakoid ATPase, including its reduction, has an interesting effect on photophosphorylation in light. It makes the rate of ATP synthesis less dependent on a high protonmotive force (Morita et al. 1983; Mills and Mitchell 1984; Junesch and Gräber 1985). This must mean that some of the light energy going into photophosphorylation is used to maintain the most active catalytic form of $\mathrm{CF}_{1}$, which must be needed for ATP synthesis as well as hydrolysis.

$\mathrm{CF} 1$, isolated from nonilluminated thylakoids, is not an active ATPase. Its $\mathrm{Ca}^{2+}$-dependent ATPase can be activated by heating to $60{ }^{\circ} \mathrm{C}$ with ATP and dithiothreiotol (DTT) added, as well as by trypsin and other proteases. Other procedures that activate the ATPase of thylakoids as well as the isolated enzyme include treatment with organic solvents (Sakurai et al. 1981; Anthon and Jagendorf 1983); detergents such as octyl glucoside (Pick and Bassilian 1981), light-dependent cleavage of the $\gamma$ subunit by trypsin (Moroney and McCarty 1982), high levels of the fungal poison tentoxin (Steele et al. 1978), and physical removal of the $\epsilon$ subunit (Richter et al. 1984). The inactive state seems to be the one that is highly controlled; disruption of structure leads to exposure of the hydrolytic ability.

During the 1960s, biochemists came to realize that enzymes often went through conformational alterations during catalysis. Inspired by P. Boyer's suggestion of possible 'conformational catalysis' for $F_{1}$, Ivan Ryrie looked for exchange of $\mathrm{H}$ atoms between medium-exposed groups on the protein and ${ }^{3} \mathrm{H}_{2} \mathrm{O}$. Up to $500 \mathrm{H}$ atoms on thylakoid-bound $\mathrm{CF}_{1}$ were found to undergo exchange if the thylakoids went into the 
high-energy state, but not in the dark or if uncouplers were present in light (Ryrie and Jagendorf 1971). In another approach, a series of chemical modifying reagents were shown, over several years, to attack $\mathrm{CF}_{1}$ if thylakoids were in light, but this did not occur in the dark. The first of these was N-ethylmaleimide, attaching to a sulfhydryl group on the $\gamma$ subunit only in light (McCarty et al. 1972; McCarty and Fagan 1973). Space does not permit a real review of this interesting area, but one outstanding study (KomatsuTakaki 1989) showed accelerated attack by pyridoxal$5^{\prime}$-phosphate on a lysine of the epsilon subunit in light.

\section{Mechanism of $\mathrm{CF}_{1}$ action: chemiosmotic concepts take over}

From the time of discovery of oxidative phosphorylation, the connection between electron flow and the chemistry of high-energy phosphate bond formation was an intriguing mystery. Essentially all of the speculation about this connection relied on the paradigm of ATP synthesis performed by triose-P dehydrogenase. This was shown to include a low-energy phosphate addition to an SH group on the enzyme, rising to a high-energy level due to oxidation. Therefore, biochemists kept looking for a high-energy intermediate, prior to ATP synthesis, involving one or more of the electron transport enzymes of mitochondria (examples in Chance and Williams 1956).

In the search for a chemical intermediate in photophosphorylation, chloroplasts were illuminated in a pipette (Shen and Shen 1962) or a syringe (Hind and Jagendorf 1963), then injected into buffer containing ${ }^{32} \mathrm{P} \mathrm{Mg}^{2+}$, and ADP, where some ATP synthesis then occurred. (The two discoveries were quite independent.) There was a problem with the results, however: the amount of ATP made in the dark was up to 50 times more than the concentration of any single electron carrier in the thylakoid membrane. Thus there must have been actual turnover of the enzymatic machinery in the dark. For a more detailed description of this, and other work from this laboratory, see Jagendorf (1998).

Earlier, based on his understanding of the physiology and physical chemistry of ion transport, Peter Mitchell (Figure 2) had proposed a drastically different idea for the mechanism. Realizing that only organelles with membranes accomplished oxidative and photosynthetic phosphorylation, he developed a 'chemiosmotic' concept of the mechanism (Mitchell 1961). In this, the connecting link is the active vec-

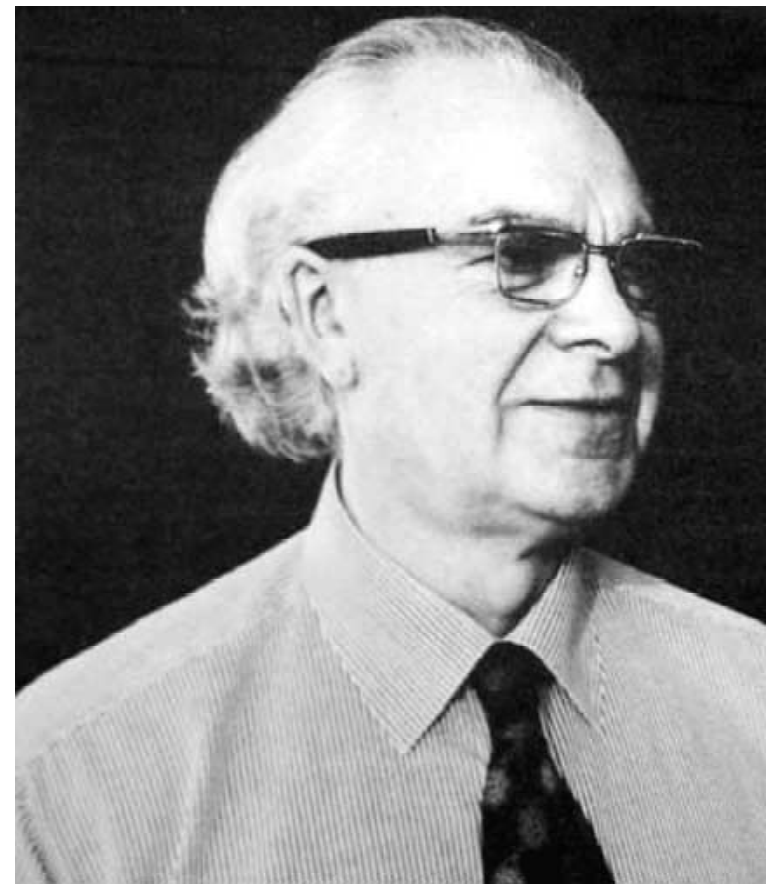

Figure 2. Peter Mitchell.

torial transport of protons across the membrane, as electron flow proceeds alternately from metals (iron or copper) handling only electrons to quinones requiring both an electron and a proton to be reduced. The highenergy intermediate, therefore, would be a difference between the electrochemical activity (both chemical concentration and membrane potential) of protons on the inside, with that on the outside of the membrane. The intermediate would drive a membrane-located, vectorial, reversible, proton-pumping ATPase.

Peter Mitchell's terminology and writing were difficult to understand. I was fortunate in having a brilliant postdoctoral from England, Geoffrey Hind (Figure 3), who could explain the concept to me. In discussing it, it occurred to us that it might be possible to observe the uptake of protons into thylakoids in light and their dissipation in the dark. It was an exciting moment to observe this happening using a simple glass electrode (Jagendorf and Hind 1963). Its relationship to photophosphorylation was further confirmed by finding that uncouplers lead to rapid dissipation of the internal protons (Neumann and Jagendorf 1964; Jagendorf and Neumann 1965).

Others realized that a massive movement of protons had to be accompanied by some sort of counterion flux to keep the membrane potentials in a reasonable range. The first counter-ion flux observed was extrusion of $\mathrm{K}^{+}$and $\mathrm{Mg}^{2+}$ in light (Dilley and Vernon 


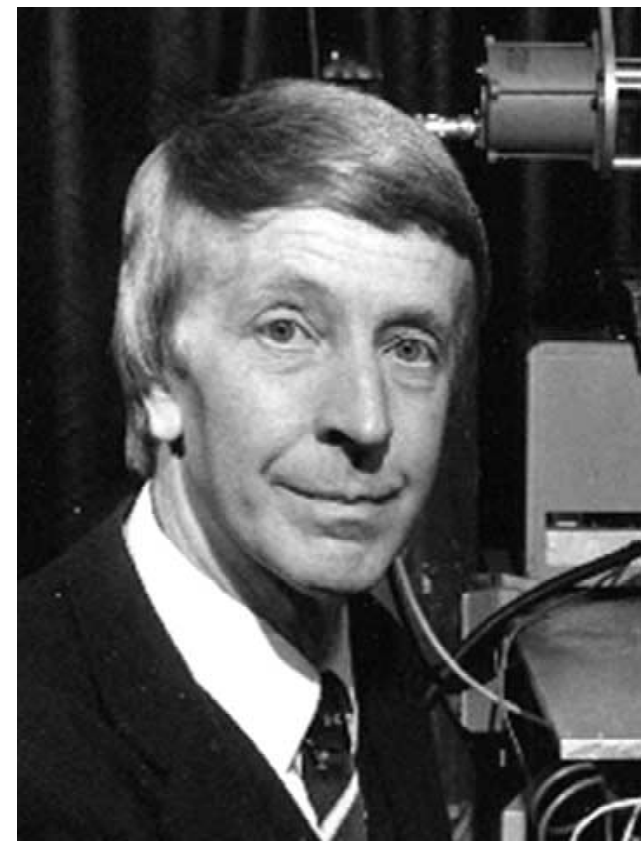

Figure 3. Geoffrey Hind.

1965). Somewhat later (Deamer and Packer 1969), the membrane potential was found to be controlled to an even greater extent by the uptake of $\mathrm{Cl}^{-}$ions with the protons. This seems to occur through a thylakoid chloride channel, discovered by Vambutus et al. (1984). A more complete discussion of ion transport is found in the review by Rottenberg (1985).

The rise in external $\mathrm{pH}$ in light implied that the $\mathrm{pH}$ of the thylakoid lumen would become more acid. Evidence for this came from the careful measurement of penetrating labeled amines and of cationic dyes in light (see Rottenberg 1985). The estimated internal $\mathrm{pH}$ dropped to $4-4.5$, as the external $\mathrm{pH}$ rose to $7.5-8$. The $3.5 \mathrm{pH}$ unit difference between external and internal spaces was certainly high enough to drive net synthesis of ATP at physiological substrate concentrations.

The first direct indication of a membrane potential was deduced from carotenoid absorption changes (the 'electrochromic' shift at $515 \mathrm{~nm}$ ), related to the coupling state of chloroplasts (Junge and Witt 1968). A similar change in bacterial 'chromatophores' was shown to serve as an intrinsic voltmeter for the membrane potential (Jackson and Crofts 1971). Other estimates come from the change in distribution of charged anions or cations into the energized organelle (see Rottenberg 1985). A full discussion is found in Junge and Jackson (1982)

\section{Acid to base induced ATP synthesis}

In trying to find spectrophotometric evidence for the energetic state, Hind found that pre-illumination of the thylakoids caused an increase in their light-scattering ability (Hind and Jagendorf 1965). This was especially true at lower $\mathrm{pH}$ levels, which had provided higher level of postillumination ATP synthesis in light (Hind and Jagendorf 1963). Looking for a correlation between light scattering and the dark ATP synthesis, Hind inserted a control in which thylakoids were never exposed to light, but simply were shifted from $\mathrm{pH} 4.5$ to $\mathrm{pH}$ 8. To our surprise, this one protocol provided a very small amount of ATP synthesis (about $10 \%$ of that seen when light had been used).

In Hind's experiments, the acid $\mathrm{pH}$ had been adjusted using $\mathrm{HCl}$. Worried about possible damage by a strong mineral acid, after Hind left I tried the same experiment using a divalent acid (phthallic) to adjust the acid $\mathrm{pH}$. This increased the amount of ATP made in darkness a great deal; and work with other dicarboxylic organic acids (succinic, maleic, etc.) increased the yield 10-fold, to twice that seen with pre-illumination (Jagendorf and Uribe 1966). This amounted to up to 100 times the level of any electron carrier in the thylakoids. The fact that ATP was being made without any electron flow was further emphasized by the failure of electron transport inhibitors to prevent the acid-base ATP synthesis, and indeed some of them were able to increase the ATP yield (Miles and Jagendorf 1970).

Other work further confirmed the validity of Mitchell's chemiosmotic hypothesis. Mitochondrial particles were shown to make ATP due to an acid/base transition (Thayer and Hinkel 1975). Most spectacular was the demonstration that photophosphorylation could be done by liposomes into which had been inserted both the light-activated, proton-pumping bacteriorhodopsin from Halobacterium halobium, and the $\mathrm{F}_{\mathrm{o}} \mathrm{F}_{1}$ complex from mitochondria (Racker and Stoeckenius 1974). There was no evolutionary precedent for the mating of those two components; so it must have been the protonmotive force generated by illuminated rhodopsin that drove the $F_{1}$ to turn over, catalyzing the synthesis of ATP.

The question was raised whether the $\mathrm{pH}$ difference or the membrane potential is most responsible for ATP synthesis. The $\mathrm{pH}$ jump-driven ATP synthesis had been done with swollen thylakoids, previously washed in $10 \mathrm{~mm} \mathrm{NaCl}$. This permitted the entry of very considerable levels of the divalent acid, which correlated well with the extent of ATP synthesis (Uribe 
and Jagendorf 1967). In the illuminated steady state, all estimates of internal $\mathrm{pH}$ showed a sufficient $\mathrm{pH}$ difference to drive ATP synthesis. Also, uncouplers (amines, nigericin, etc.) which collapse only the $\mathrm{pH}$ difference stop ATP synthesis.

The membrane potential, on the other hand, is the major force with very small subchloroplast particles. There, valinomycin, collapsing the membrane potential but not a $\mathrm{pH}$ gradient, inhibited ATP synthesis (McCarty 1969). Also, in flashing light, where protons move in and insufficient time is given for counter-ion flux to neutralize the electric charge, valinomycin, but not uncouplers such as amines, inhibit ATP formation [see Rumberg (1977) for a discussion].

The most spectacular demonstration of the role of membrane potential seemed to occur in experiments by H.T. Witt and colleagues. In these, an applied voltage in the medium was found to drive ATP synthesis (Witt et al. 1976). However, the interpretation of the results was questioned when it was determined, later, that too much ATP had been formed; perhaps the electric pulse had caused internal accumulation of a small proton pool [see Rottenberg (1985) for a discussion].

In addition to the distinction between proton gradient and membrane potential components, the exclusive function of internal bulk phase protons as the energetic intermediate ('delocalized coupling') has been questioned. Various discrepancies suggested the alternative operation of a close, more direct connection between the proton pumps and the ATPase complex ('localized' coupling). Evidence from R. Dilley's laboratory indicates alternative operation of either of these driving ATP synthesis, depending on the ionic composition of the medium, especially the $\mathrm{Ca}^{2+}$ concentration. These ideas are discussed in a review (Dilley 1991). The physiological significance of the two forms is not yet clear.

Much effort has gone into attempts at determining the number of protons pumped per electron moving through the chain, and the number of protons needed to form one ATP. These are both difficult to measure accurately, because of the turnover of internal protons. While most early efforts indicated a proton:ATP ratio of 3, something like a consensus appeared later (Van Walraven et al. 1996) that the number should be 4 .

\section{Contribution of the chemiosmotic theory to photosynthesis}

The chemiosmotic interpretation helps explain several other photosynthetic details. First of all, the rise in $\mathrm{pH}$ of the stroma as protons are moved into the lumen under light, together with the movement of $\mathrm{Mg}^{2+}$ into the stroma, provide conditions much more favorable for operation of the $\mathrm{CO}_{2}$ fixing cycle. Estimates have been made that the stroma $\mathrm{pH}$ rises from 7.1 in the dark to 7.8 or 8 in the light (Buchanan 1980).

With protonmotive force as the intermediate between electron flow and ATP synthesis, degrading the proton electrochemical activity gradient will obviously inhibit photophosphorylation. That explains the uncoupling action of detergents (Neumann and Jagendorf 1965), which permit leakage of protons; ionophores like nigericin that exchange $\mathrm{H}^{+}$for $\mathrm{K}^{+}$; and of $\mathrm{NH}_{3}$ and amines, which neutralize the internal acidity.

It also clarifies the nature of the coupling 'sites' in the electron transport chain, as regions where protons are caused to move across the thylakoid membrane. For thylakoids, there are two such natural sites. One is in the oxidation of 2 moles of $\mathrm{H}_{2} \mathrm{O}$ in Photosystem II, PS II (Schwarz 1968; Ouitrakul and Izawa 1973; Trebst and Reimer 1973). The enzymes are located on the lumenal side of the thylakoid, and, therefore both $\mathrm{O}_{2}$, and four $\mathrm{H}^{+}$are released into the lumen.

The second is in the transfer from reduced plastoquinone to the cytochrome $b_{6} / f$ complex, between the two photosystems. Here, the quinone(s) reduced by PS II pass only their electrons to the cytochromes; the $\mathrm{H}^{+}$associated is released into the lumen. In addition, a complex pattern can occur in the $b_{6} / f$ complex, which has different quinone-binding sites on the internal and external faces of the thylakoid. Designated the 'Q cycle' (Mitchell 1975), some of the electrons move on to PS I, but others are recycled back to the quinone pool through the $b$ type cytochromes, resulting in a transfer of an extra proton to the interior of the thylakoid. Critical evidence for its occurrence in chloroplasts has been difficult to obtain, but there were many indirect indications (one example-a high $\mathrm{H}^{+} / \mathrm{e}^{-}$ ratio-Kobayashi et al. 1995). Recent structural analysis of the $b_{6} / f$ complex supports it as well (Carrell et al. 1997).

The other major pattern for electron flow is the cycle around PS I. In that case, the electron coming from PS I is transmitted, through ferredoxin in the stroma (Tagawa et al. 1963) to the external quinone component of the cytochrome $b_{6} / f$ complex. This 
quinone has to pick up a proton, at the same time, from the stroma. Redox shuffling through the complex must go through a quinone on the lumenal side; and when it is oxidized its proton remains inside. Cyclic electron flow through ferredoxin has the unique characteristic for chloroplasts of being inhibited by antimycin A (Tagawa et al. 1963).

A simpler system is the one using one of the artificial dyes, methyl phenazinium sulfate or its derivative, pyocyanine. These have been shown (Hauska et al. 1974, 1975) to interact with nothing but PS I. They take an electron from PS I on the outside, pick up a proton from the medium, then move into the lumen where they are oxidized by PS I, releasing the proton to the bulk phase.

\section{Further developments}

Enormously important findings, and changing concepts, have occurred since the chemiosmotic concept took over. Space permits only very brief mention of some of these.

A revolutionary new idea came from Boyer's finding that synthesis of the high-energy phosphate bond of ATP does not need any energy input, when the substrates are bound on the $\mathrm{F}_{1}$ or $\mathrm{CF}_{1}$. Energy is needed to release ATP from its binding site, and in lesser amount to add ADP and $P_{i}$ to theirs [see Shavit et al. (1967) for the finding with chloroplasts].

A truly important new concept was the 'binding change' hypothesis, (i.e. site-site cooperativity), developed by Paul Boyer again, from $\mathrm{O}$ exchange data [see Shavit et al. (1967)), Stroop and Boyer (1985) for findings with $\mathrm{CF}_{1}$ ]. This means that the newly formed ATP is not released until a new ADP binds to an adjacent site. The input of energy then releases ATP from the first site, and tightens the binding of ADP and $\mathrm{P}_{\mathrm{i}}$ at the second, so that they, in turn, can catalyze the freely reversible reaction of ADP $+\mathrm{P}_{\mathrm{i}} \Longleftrightarrow \mathrm{ATP}+\mathrm{H}_{2} \mathrm{O}$.

While the three $\beta$ subunits are identical, during catalysis they must have alternative configurations, both because of the alternative nucleotide binding condition, and because there is only one $\gamma$ subunit. This led to the imaginative concept that during catalysis the $\gamma$ subunit must touch each one of the $\beta$ subunits in turn. To do so, it would have to rotate within the center of the complex. This physical rotation of $\gamma$ has been identified in a remarkable series of papers reviewed most recently by Yoshida et al. (2001).

\section{Acknowledgment}

This manuscript was prepared with the aid of a Liberty Hyde Bailey professorship from Cornell University.

\section{References}

Allen MB, Whatley FR and Arnon DJ (1958) Photosynthesis by isolated chloroplasts. VI. Rates of conversion of light into chemical energy in photosynthetic phosphorylation. Biochim Biophys Acta 27: 16-23

Anthon GE and Jagendorf AT (1983) Effect of methanol on spinach thylakoid ATPase. Biochim Biophys Acta 723: 358-365

Arnon DI, Allen MB and Whatley FR (1954) Photosynthesis by isolated chloroplasts. II. Photophosphorylation, the conversion of light into phosphate bond energy. J Am Chem Soc 76: 6324-6329

Arnon DI, Whatley FR and Allen MB (1958a) Assimilatory power in photosynthesis. Science 127: 1026-1034

Arnon DI, Whatley FR and Allen MB (1958b) Photosynthesis by isolated chloroplasts. VIII. Photosynthetic preparation and the generation of assimilatory power. Biochim Biophys Acta 32: $47-57$

Arnon DI, Losada M, Whatley FR, Tsujimoto HY, Hall DO and Horton AA (1961) Photosynthetic phosphorylation and molecular oxygen. Proc Natl Acad Sci USA 47: 1314-1344.

Avron M (1963) A coupling factor in photophosphorylation. Biochim Biophys Acta 77: 699-702

Avron M and Jagendorf AT (1958) Co-factors and rates of photosynthetic phosphorylation by spinach chloroplasts. J Biol Chem 231: 277-290

Avron M and Jagendorf AT (1959) Evidence concerning the mechanism of ATP formation by spinach chloroplasts. J Biol Chem 234: 967-972

Avron M, Krogmann DW and Jagendorf AT (1958) The relation of photosynthetic phosphorylation to the Hill reaction. Biochim Biophys Acta 30: 144-153

Bakker-Grunwald T (1977) ATPase. In: Trebst A and Avron M (eds) Encyclopedia of Plant Physiology, New Series, Vol 5, pp 369373. Springer-Verlag, Berlin

Buchanan BB (1980) Role of light in the regulation of chloroplast enzymes. Annu Rev Plant Physiol 31: 341-374

Carmeli C and Racker E (1973) Partial resolution of enzymes catalyzing photophosphorylation. XIV. Reconstitution of chlorophyll-deficient vesicles catalyzing phosphate-adenosine triphosphate exchange. J Biol Chem 248: 8281-8287

Carrell CJ, Zhang H, Cramer WA and Smith JL (1997) Biological identity and diversity in photosynthesis and respiration: structure of the lumen-side domain of the chloroplast Rieske protein. Structure 5: 1613-1625

Chance B and Williams CR (1956) The respiratory chain and oxidative photophosphorylation. Adv Enzymol 17:65-134

Deamer DW and Packer L (1969) Light-dependent anion transport in isolated spinach chloroplasts. Biochim Biophys Acta 172: 539

Dilley RA (1991) Energy coupling in chloroplasts: a calcium-gated switch controls proton fluxes between localized and delocalized proton gradients. Curr Topics Bioenerg 16: 265-318

Dilley RA and Vernon LP (1965) Ion and water transport processes related to the light-dependent shrinkage of spinach chloroplasts. Arch Biochem Biophys 111: 365-373

Emerson R, Stauffer JS and Umbreit WW (1944) Relationships between photosynthesis and phosphorylation in Chlorella. Am J Bot 31: 107-120 
Farron F (1970) Isolation and properties of a chloroplast coupling factor and heat-activated ATPase. Biochemistry 9: 3823-3828

Frenkel A (1954) Light-induced phosphorylation by cell-free preparations of photosynthetic bacteria. J Am Chem Soc 76: 55685569

Futai M, Noumi T and Maeda M (1989) ATP synthase $\left(\mathrm{H}^{+}\right.$. ATPase): results by combined biochemical and molecular biological approaches. Annu Rev Biochem 58: 111-136

Gest H and Kamen MD (1948) Studies on the phosphorus metabolism of green algae and purple bacteria in relation to photosynthesis. J Biol Chem 176: 299-318

Good NE (1960) Activation of the Hill reaction by amines. Biochim Biophys Acta 40: 502-517

Hauska G, Reimer S and Trebst A (1974) Native and artificial energy-conserving sites in cyclic photophosphorylation systems. Biochim Biophys Acta 357: 1-13

Hauska G, Oettmeier W, Reimer S and Trebst A (1975) Energy conservation in photoreductions by Photosystem I. Shuttles of artificial electron donors for Photosystem I across the thylakoid membrane. Z Naturforsch C 30: 37-45

Hightower KE and McCarty RE (1996) Influence of nucleotides on the cold stability of chloroplast coupling factor 1. Biochemistry 35: 10051-10057

Hind G and Jagendorf AT (1963) Separation of light and dark stages in photophosphorylation. Proc Nat Acad Sci USA 49: 715-722

Hind G and Jagendorf AT (1965) Light scattering changes associated with the production of a possible intermediate in photophosphorylation. J Biol Chem 240: 3195-3201

Jackson JB and Crofts AR (1971) The kinetics of light induced carotenoid changes in Rhodopseudomonas spheroides and their relation to electrical field generation across the chromatophore membrane. Eur J Biochem 18: 120-130

Jagendorf AT (1962) Biochemistry of energy transformations during photosynthesis. In: Glass HB (ed) Survey of Biological Progress, Vol IV, pp 181-344. Academic Press, New York

Jagendorf AT (1998) Chance, luck and photosynthesis research - an inside story. Photosynthesis Res 57: 215-229

Jagendorf AT and Hind G (1963) Studies on the mechanism of photophosphorylation, In: Kok B and Jagendorf AT (eds) Photosynthetic Mechanisms of Green Plants, pp 599-610. Publication 1145 of National Academy of Sciences-National Research Council, Washington, DC

Jagendorf AT and Neumann J (1965) Effect of uncouplers on the light-induced $\mathrm{pH}$ rise with spinach chloroplasts. J Biol Chem 240: 3210-3214

Jagendorf AT and Smith M (1962) Uncoupling phosphorylation in spinach chloroplasts by absence of cations. Plant Physiol 37: 135-141

Jagendorf AT and Uribe E (1966) ATP formation caused by acidbase transition of spinach chloroplasts. Proc Nat Acad Sci USA 55: $170-177$

Jagendorf AT, McCarty RE and Robertson D (1991) Coupling factor components: structure and function. In: Bogorad L and Vasil IK (eds) Cell Culture and Somatic Cell Genetics of Plants, Vol 7B, pp 225-254. Academic Press, New York

Junesch U and Gräber P (1985) The rate of ATP synthesis as a function of $\mathrm{DpH}$ in normal and dithiothreitol-modified chloroplasts. Biochim Biophys Acta 809: 429-434

Junge W and Jackson JB (1982) The development of electrochemical potential gradients across photosynthetic membranes. In: Govindjee (ed) Photosynthesis: Energy Conversion by Plants and Bacteria, pp 589-646. Academic Press, New York

Junge W and Witt HT (1968) On the ion transport system of photosynthesis. Investigations on a molecular level. Z Naturforsch 23b: $244-254$
Kamienietzky A and Nelson N (1975) Preparation and properties of chloroplasts depleted of chloroplast coupling factor 1 by sodium bromide treatment. Plant Physiol 55: 282-287

Kandler O (1950) Über die Beziehungen zwischen Phosphathaushalt und Photosynthese. I. Phosphatspiegelschwenkungen bei Chlorella pyrenoidosa als Folde des Licht-Dunkel-Wechsels. Z Naturforsch 5b: 423-437

Kobayashi Y, Neimanis S and Heber U (1995) Coupling ration $\mathrm{H}^{+} / \mathrm{e}^{-}=3 \mathrm{vs} \mathrm{H}^{+} / \mathrm{e}^{-}=2$ in chloroplasts, and quantum requirements of oxygen exchange during the reduction of nitrite, ferricyanide or methylviologen. Plant Cell Physiol 6: 1613-1620

Komatsu-Takaki M (1989) Energy-dependent conformational changes in the subunit of the chloroplast ATP synthase $\left(\mathrm{CF}_{\mathrm{o}} \mathrm{CF}_{1}\right)$. J Biol Chem 264: 17750-17753

Krogmann DW, Jagendorf AT and Avron A (1959) Uncouplers of spinach chloroplast photosynthetic phosphorylation. Plant Physiol 34: 272-277

Lardy HA and Wellman H (1952) Oxidative phosphorylations: role of inorganic phosphate and acceptorsystems in control of metabolic rates. J Biol Chem195: 215-224

Lien S and Racker E (1971) Partial resolution of the enzymes catalyzing photophosphorylation. VIII Properties of silico-tungstate treated particles. J Biol Chem 246: 4298-4307

Loomis WF and Lipmann F (1948) Reversible inhibition of the coupling between phosphorylation and oxidation. J Biol Chem 173: 807-808

Maclachlan GA and Porter HK (1959) Replacement of oxygen by light as the energy source for glucose metabolism in tobacco leaves. Proc Roy Soc London B 150: 460-473

McCarty RE (1969) The uncoupling of photophosphorylation by valinomycin and ammonium chloride. J Biol Chem 244: 42924298

McCarty RE (1992) A plant biochemist's view of $\mathrm{H}^{+}$-ATPases and ATP synthases. J Exp Bot 172: 431-441

McCarty RE and Fagan J (1973) Light-stimulated incorporation of $\mathrm{N}$-ethylmaleimide into coupling factor 1 in spinach chloroplasts. Biochem 12: 1503-1507

McCarty RE, Pittman PR and Tsuchiya Y (1972) Light-dependent inhibition of photophosphorylation by N-ethylmaleimide. J Biol Chem 247: 3048-3051

Mehler AH (1951) Studies on reactions of illuminated chloroplasts. I. Mechanism of the reduction of oxygen and other Hill reagents. Arch Biochem Biophys 33: 65-77

Miles CD and Jagendorf AT (1970) Evaluation of electron transport as the basis of ATP synthesis after acid-base transition by spinach chloroplasts. Biochemistry 9: 429-4341

Mills JD and Mitchell P (1984) Thiol modulation of the chloroplast protonmotive ATPase and its effect on photophosphorylation. Biochim Biophys Acta 764: 93-104

Mitchell P (1961) Coupling of phosphorylation to electron and hydrogen transfer by a chemiosmotic type of mechanism. Nature (London) 191: 144-148

Mitchell P (1975) The proton-motive Q cycle: a general formulation. FEBS Lett 59: 137-139

Morita S, Itoh S and Nishimura M (1983) Flash-induced photophosphorylation in chloroplasts with activated ATPase. Biochim Biophys Acta 724: 411-415

Moroney and McCarty RE (1982) Light-dependent cleavage of the $\mathrm{g}$ subunit of coupling factor 1 by trypsin causes activation of $\mathrm{Mg}^{2+}$ ATPase activity and uncoupling of photophosphorylation in spinach chloroplasts. J Biol Chem 257: 5915-5920

Moroney JV, Lopresti L, McEwen BF, McCarty RE and Hammes GG (1983) The Mr value of chloroplast coupling factor 1. FEBS Lett 158: 58-62 
Nelson N, Eytan E, Notsani B, Sigrist H, Sigrist-Nelson $\mathrm{K}$ and Gitler C (1977) Isolation of a chloroplast N,N'dicyclohexylcarbodiimide-binding proteolipid, active in proton translocation. Proc Natl Acad Sci USA 74: 2375-2378

Neumann J and Jagendorf AT (1964) Light-induced pH changes related to phosphorylation by chloroplasts. Arch Biochem Biophys 107: 109-119

Ouitrakul R and Izawa S (1973) Electron transport and photophosphorylation in chloroplasts as a function of the electron acceptor. I. A receptor-specific inhibition by $\mathrm{KCN}$. Biochim Biophys Acta 305: 105-118

Petrack B and Lipmann F (1961) Photophosphorylation and photohydrolysis in cell-free preparations of blue-green algae. In: McElroy WD and Glass HB (eds) Light and Life, pp 621-630. Johns Hopins Press, Baltimore

Pick U and Bassilian S (1981) Octyl glucoside stimulates a $\mathrm{Mg}^{2+}$. specific ATPase activity in chloroplast $\mathrm{CF}_{1}$. In: Selman BR and Selman-Reimer S (eds) Energy Coupling in Photosynthesis, pp 251-260. Elsevier/North Holland, Amsterdam

Racker E (1970) Function and structure of the inner membranes of mitochondria and chloroplasts. In: Racker E (ed) Membranes of Mitochondria and Chloroplasts, pp 127-171. Van Nostrand Reinhold, New York

Racker E and Stoeckenius W (1974) Reconstitution of purple membrane vesicles catalyzing light driven proton uptake and adenosine triphosphate formation. Biochemistry 20: 662-663

Racker E, Hauska GA, Lien S, Berzborn RG and Nelson N (1972) Resolution and reconstitution of the system of photophosphorylation. In: Forti G (ed) Proceedings of the 2nd International Congress on Photosynthesis Research, Vol III, pp 1097-1112. Dr W Junk Publishers, The Hague

Richter ML, Patrie WJ and McCarty RE (1984) Preparation of the e subunit and e-deficient chloroplast coupling factor 1 in reconstitutively active forms. J Biol Chem 259: 7371-7373

Rottenberg H (1985) Proton-coupled energy conversion: chemiosmotic and intramembrane coupling. Modern Cell Biol. 4: 47-83

Ruben S (1943) Photosynthesis and phosphorylation. J Am Chem Soc 65: 279-282

Rumberg B (1977) Field Changes. In: Trebst A and Avron M (eds) Encyclopedia of Plant Physiology, New Series, Vol 5, pp 405415. Springer-Verlag, Berlin

Ryrie I and Jagendorf AT (1971) An energy-linked conformational change in the coupling factor protein in chloroplasts. Studies with hydrogen exchange. J Biol Chem 246: 3771-3774

Sakurai H, Shinohara K, Hisabori T and Shinohara K (1981) Enhancement of adenosine triphosphatase activity of purified chloroplast coupling factor 1. J Biochem (Tokyo) 90: 95-102

Schwartz M (1968) Light induced proton gradient links electron transport and photophosphorylation. Nature (London) 219: 915919

Schwink L (1956) Nachweis von Adenosinetriphosphosauer (ATP) in Grünalgen und Helodea sowie Einbau von radioaktivem Phosphor $\left({ }^{32} \mathrm{P}\right)$ bei der Photosynthese. Planta 47: 165-218

Sebald W and Hoppe J (1981) On the structure and genetics of the proteolipid subunit of the ATP synthase complex. In: Sanadi DR (ed) Current Topics in Bioenergetics, Vol 12, pp 1-64. Academic Press, New York
Shavit N, Skye GE and Boyer PD (1967) Occurrence and possible mechanism of ${ }^{32} \mathrm{P}$ and ${ }^{18} \mathrm{O}$ exchange reactions of photophosphorylation. J Biol Chem 242: 5125-5130

Shen YK and Shen GM (1962) The 'light intensity effect' and intermediate steps of photophosphorylation. Scientia Sinica 11: 1097-1106

Simonis W and Grube KH (1952) Untersuchungen über den Zusammenhang von Phosphathaushalt und Photosynthese. Z Naturforsch 7b: 194-196

Steele JA, Uchytil RF and Durbin RB (1978) The stimulation of coupling factor 1 ATPase by tentoxin. Biochim Biophys Acta 504: 136-141

Strehler BL (1953) Firefly luminescence in the study of energy transfer mechanisms. II. Adenosine triphosphate and photosynthesis. Arch Biochem Biophys 43: 67-79

Stroop SD and Boyer PD (1985) Characteristics of the ATP synthase as revealed by reaction at low ADP concentrations. Biochem 24: 2304-2310

Tagawa K, Tsujimoto HY and Arnon DI (1963) Role of chloroplast ferredoxin in the energy conversion process of photosynthesis. Proc Natl Acad Sci USA 49: 567-572

Telfer A, Barber J and Jagendorf AT (1980) Electrostatic control of chloroplast coupling factor binding to thylakoid membranes as indicated by cation effects on electron transport and reconstitution of photophosphorylation Biochim Biophys Acta 591: 331-345

Thayer WS and Hinkle PC (1975) Synthesis of adenosine triphosphate by an artificially imposed electrochemical proton gradient in bovine heart submitochondrial particles. J Biol Chem 250: 5330-5335

Trebst A and Reimer S (1973) Properties of photoreductions by Photosystem II in isolated chloroplasts. An energy-conserving step in the photoreduction of benzoquinone by Photosystem II in the presence of dibromothymoquinone. Biochim Biophys Acta 305: 129-139

Uribe E and Jagendorf AT (1967) On the localization of organic acids in acid-induced ATP synthesis. Plant Physiol 42: 697-705

Vambutas VK and Racker E (1965) Partial resolution of the enzymes catalyzing photophosphorylation. I. Stimulation of photophosphorylation by a preparation of a latent, $\mathrm{Ca}^{2+}$-dependent adenosine triphosphatase from chloroplasts. J Biol Chem 240: 2660-2667

Vambutas V, Beattie DS and Bittman R (1984) Isolation of chloride ion transport active protein(s) from thylakoid membranes. Arch Biochem Biophys 232: 538-548

Van Walraven HS, Strotmann H, Schwarz O and Rumberg B (1996) The $\mathrm{H}^{+} /$ATP coupling ratio of the ATP synthase from thiolmodulated chloroplasts and two cyanobacterial strains is four. FEBS Lett 379: 309-313

Wassink EC (1957) Phosphate in the photosynthetic cycle in Chlorella. In: Gaffron H, Brown AH, French CS, Livingston R, Rabinowitch E, Strehler BL and Tolbert NE (eds) Research in Photosynthesis, pp 333-339. Interscience, New York

Williams AM (1956) Light-induced uptake of inorganic phosphate in cell-free extracts of obligately anaerobic photosynthetic bacteria. Biochim Biophys Acta 19: 570

Yoshida M, Muneyukis E and Hisabori T (2001) ATP synthase a marvelous rotary engine of the cell. Nature Rev Mol Biol 2 669-677 